\title{
Back Propagation Neural Network for Controlling Coupled Water Tank
}

\author{
Halim Mudia \\ Department of Electrical Engineering, State Islamic University of Sultan Syarif Kasim Riau \\ Email: halim.mudia@uin-suska.ac.id
}

\begin{tabular}{|c|c|}
\hline Article Info & ABSTRACT \\
\hline Article history: & \multirow{9}{*}{$\begin{array}{l}\text { A well-prepared abstract enables the reader to identify the basic content of the } \\
\text { level and flow control in tanks is the heart of all chemical engineering } \\
\text { systems. The control of liquid level in tanks and flow between tanks is } \\
\text { a basic problem in the process industries. Many times the liquids will } \\
\text { be processed by chemical or mixing treatment in the tanks, but always } \\
\text { the level of fluid in the tanks must be controlled and the flow between } \\
\text { tanks must be regulated in presence of non-linearity. Therefore, in this } \\
\text { paper will use neural network based on backpropagation (BP) to } \\
\text { control of level } 2 \text { in the tank } 2 \text { with the setpoint of } 10 \text { centimeters and } \\
\text { can follow the setpoint changes to } 8 \text { centimeters given in } 225 \text { seconds. } \\
\text { The results show that a neural network based on backpropagation can } \\
\text { follow setpoint given with steady-state error is } 0 \text { cm, overshoot is } 0 \% \text {, } \\
\text { the rising time is } 49 \text { seconds, settling time is } 52 \text { seconds and can follow } \\
\text { setpoint changes in } 51 \text { seconds. }\end{array}$} \\
\hline Received May 02, 2020 & \\
\hline Revised May 24, 2020 & \\
\hline Accepted June 06, 2020 & \\
\hline Keywords: & \\
\hline Backpropagation & \\
\hline Coupled Water Tank & \\
\hline Level Control & \\
\hline Neural Network & \\
\hline
\end{tabular}

This is an open access article under the CC BY-SA license.

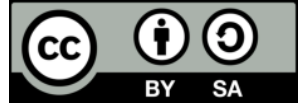

\section{Corresponding Author:}

Halim Mudia

Department of Electrical Engineering Faculty of Science and Technology

State Islamic University of Sultan Syarif Kasim Riau

Soebrantas 155 Pekanbaru - Indonesia

Email: halim.mudia@uin-suska.ac.id

\section{INTRODUCTION}

Neural Networks (NNs) are a set of powerful mathematical tools that simulate the way that the human brain deals with information and the procedure of learning. NNs have the ability to identify and learn highly complex and nonlinear relationships from input-output data only, without the use of first principle equations describing the system. Neural networks are categorized to a variety of architectures, depending on the way the nodes of the neural network are interconnected and the calculations that each node performs [1].

The backpropagation (BP) neural networks have been widely used in many applications [2], [3]. Their strong ability to approximate nonlinear mappings is discussed in, e.g., [4] and [5]. There has been much work on the convergence of the training iteration procedure for BP neural networks by using the gradient method [6]-[7], [8]. For more advanced learning methods and other related discussions, we refer to [9], [10], [11], and [12]. There are two practical ways in the implementation of the gradient method: batch learning and online learning. The batch learning corresponds to the standard gradient method, where the network weights are updated, each time in the iteration procedure of the learning after all the training examples are processed by the network. Online learning is a variation of the standard gradient method, where the network weights are updated after each training example is processed. In engineering computation of neural networks, the online gradient method is commonly used due to its simplicity and efficiency [13], [14], [15]. The convergence of this method for the linear case has been studied in, e.g., [16] and [17] among many others. For the nonlinear case the existing 
convergence results [6], [18], [19] are mostly concerned with the asymptotic convergence with a probabilistic nature as the size of the training examples goes to infinity. Convergence results of deterministic nature are given in [20] and [21]-[22], for a feedforward network without a hidden layer. It is required in [21] that the training examples are linearly independent, which is a rather restrictive condition since the number of training examples in practice is often very large and inevitably they are linearly dependent.

The liquid level control in Coupled Tank System is a classical benchmark control problem. Level control is one of the control system variables which are more important in process industries. The process industries require liquid to be pumped as well as stored in tanks and then repumped to another tank. Many times the liquids will be processed by chemical or mixing treatment in the tanks, but always the level of fluid in the tanks must be controlled and the flow between tanks must be regulated. The quality of the product of the mixture depends on the level of the reactants in the mixing tank. Tank level control systems are used frequently in different processes. All of the pharmaceutical industries, petrochemical plants, food industries, and nuclear power plants depend upon tank level control systems. Control system engineers need to understand how to tank control systems work and how the level control problem is solved. The liquid level system has a time varying system parameters and non-linear characteristics in the complex industrial process. Most of the control performances in the actual design are usually defined by steady-state error, overshoot, rising time, settling time [22].

Various attempts in controlling the liquid level of the coupled tank system were proposed. The design of the PI controller using characteristics ratio assignment method for linear modeled coupled tank SISO process was proposed by various attempts in controlling the liquid level of the coupled tank system was proposed. Various attempts in controlling the liquid level of the coupled tank system were proposed. The design of PI controller using characteristics ratio assignment method for linear modeled coupled tank SISO process was proposed by M. Senthilkumar et al [23] and The mathematical modeling and designing of sliding mode control for a liquid level control system when tanks are coupled by using baffles were proposed by Hur Abbas et al [24]. Muhammad Nasiruddin Mahyuddin et al proposed a Direct model reference adaptive control for coupled tank system [25], Comparison between PI and MRAC on coupled tank system done by M. Saad [26] and Comparative study of Mamdani-type and Sugeno-type fuzzy inference systems for coupled water tank by Halim Mudia[27].

\section{RESEARCH METHOD}

The research method in this paper presents the mathematical modeling of the coupled water tank and design of the backpropagation neural network control for the coupled water tank.

\subsection{Mathematical Modelling Of Coupled Water Tank}

The coupled water tank as shown in Figure 1 is a coupled tank for the experimental scale. Consists of two tanks and pumps that function to pump water vertically [27]:

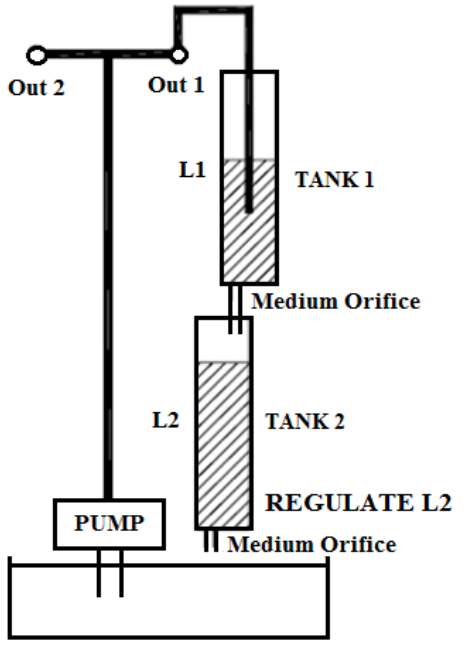

Figure 1. The Coupled Water Tank [27]

The flow into the tank 1 is:

$$
F_{1 \text { in }}=K_{m} V_{p} \mathrm{~cm}^{3} / \mathrm{sec}
$$


where $K_{m}$ is the constant pump and $V_{p}$ is the voltage applied to the pump. The outflow velocity is given by the Bernoulli equation for small orifices:

$$
V_{o}=\sqrt{2 g L_{1}} \mathrm{~cm} / \mathrm{sec}
$$

where $g$ is the gravitational acceleration in $\mathrm{cm} / \mathrm{sec}^{2}$ and $L_{1}$ is the high of the water level in the tank $1 \mathrm{in} \mathrm{cm}$. The outflow rate is:

$$
F_{1 \text { in- }} F_{1 o u t}=K_{m} V_{p}-\alpha_{1} \sqrt{2 g L_{1}} \mathrm{~cm}^{3} / \mathrm{sec}
$$

Then the change in level of tank 1 is then given, where $A_{1}$ is the diameter of the tank 1 :

$$
\dot{L}_{1}=-\frac{\alpha_{1}}{A_{1}} \sqrt{\frac{g}{2 L_{10}}} L_{1}+\frac{K_{m}}{A_{1}} V_{p}
$$

The equation of tank 2 for inflows and outflows is:

$$
\begin{aligned}
& F_{1 \text { in }}=\alpha_{1} \sqrt{2 g L_{1}} \mathrm{~cm}^{3} / \mathrm{sec} \\
& F_{2 o u t}=\alpha_{2} \sqrt{2 g L_{2}} \mathrm{~cm}^{3} / \mathrm{sec}
\end{aligned}
$$

Then the change in level of tank 2 is then given by:

$$
\dot{L}_{2}=-\frac{\alpha_{2}}{A_{2}} \sqrt{\frac{g}{2 L_{20}}} L_{2}+\frac{\alpha_{1}}{A_{2}} \sqrt{\frac{g}{2 L_{10}}} L_{1}
$$

If $x_{1}=L_{1}$ and $x_{2}=L_{2}$ then equation state of system can write as:

$$
\begin{aligned}
& \dot{x}_{1}=-\frac{\alpha_{1}}{A_{1}} \sqrt{\frac{g}{2 L_{10}}} x_{1}+\frac{K_{m}}{A_{1}} V_{p} \\
& \dot{x}_{2}=-\frac{\alpha_{2}}{A_{2}} \sqrt{\frac{g}{2 L_{20}}} x_{2}+\frac{\alpha_{1}}{A_{2}} \sqrt{\frac{g}{2 L_{10}}} x_{1}
\end{aligned}
$$

and the parameter of equation (8) and (9), can we see in table 1:

Table 1. Parameter of Coupled Water Tank [27]

\begin{tabular}{cccc}
\hline Parameter & Symbol & Value & Units \\
\hline Diameter of Tank 1 & $\mathrm{A}_{1}$ & 15,5179 & $\mathrm{~cm}^{2}$ \\
Diameter of Tank 2 & $\mathrm{A}_{2}$ & 15,5179 & $\mathrm{~cm}^{2}$ \\
Gravity & $\mathrm{g}$ & 980 & \\
Pump Constant & $\mathrm{Km}$ & 4,6 & $\left(\mathrm{~cm}^{3} / \mathrm{s}\right) /$ Volt \\
Level Water of Tank 1 & $\mathrm{L}_{2}$ & terukur & $\mathrm{cm}$ \\
Level Water of Tank 2 & $\mathrm{L}_{1}$ & terukur & $\mathrm{cm}$ \\
Cross Section Area of Tank 1 & $\alpha_{1}$ & 0,17813919765 & $\mathrm{~cm}$ \\
Cross Section Area of Tank 2 & $\alpha_{2}$ & 0,17813919765 & $\mathrm{~cm}$ \\
Pump Voltage (max) & $\mathrm{V}_{\mathrm{p}}$ & 22 & $\mathrm{Volt}$ \\
Tank 1 Work Point & $\mathrm{L}_{10}$ & 15 & $\mathrm{~cm}$ \\
Tank 2 Work Point & $\mathrm{L}_{20}$ & 15 & $\mathrm{~cm}$ \\
\hline
\end{tabular}

\subsection{Design of Backpropagation Neural Network Control for Coupled Water Tank}

In this paper we can explain 2 main parts of this design system to control the level of the Coupled Water Tank, where the setpoint is 10 centimeters and the setpoint will change to 8 centimeters in 225 seconds:

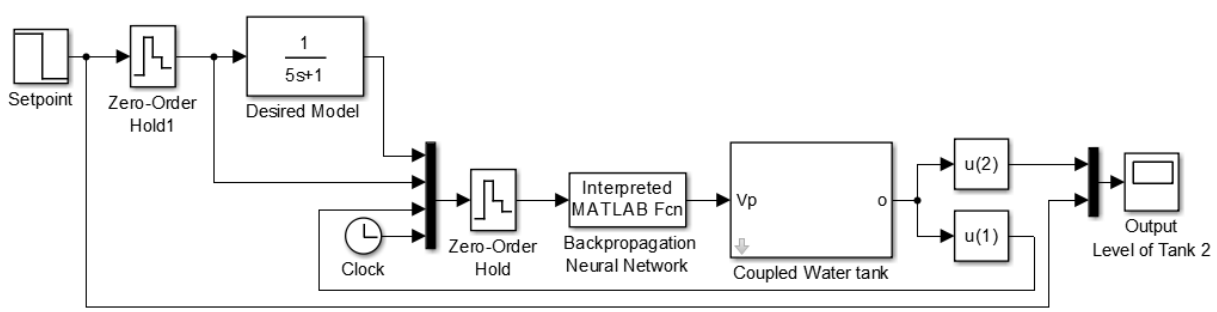

Figure 2. Design of The Coupled Water Tank and Backprogation Neural Network in Matlab 


\subsubsection{Design of Couple Water Tank:}

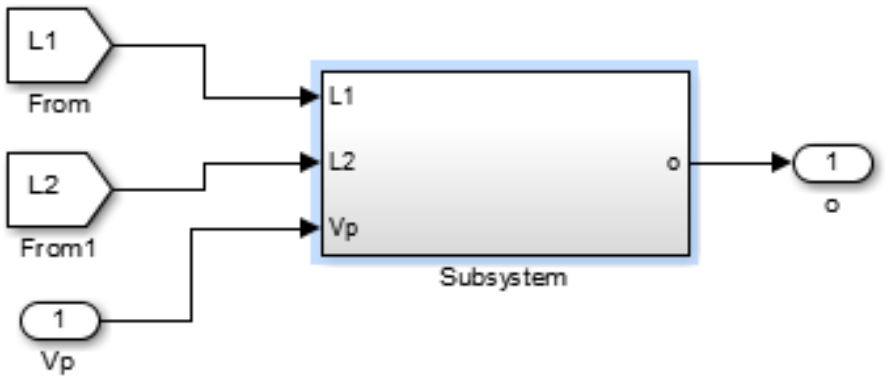

Figure 3. Design of Subsystem of The Coupled Water Tank in Matlab

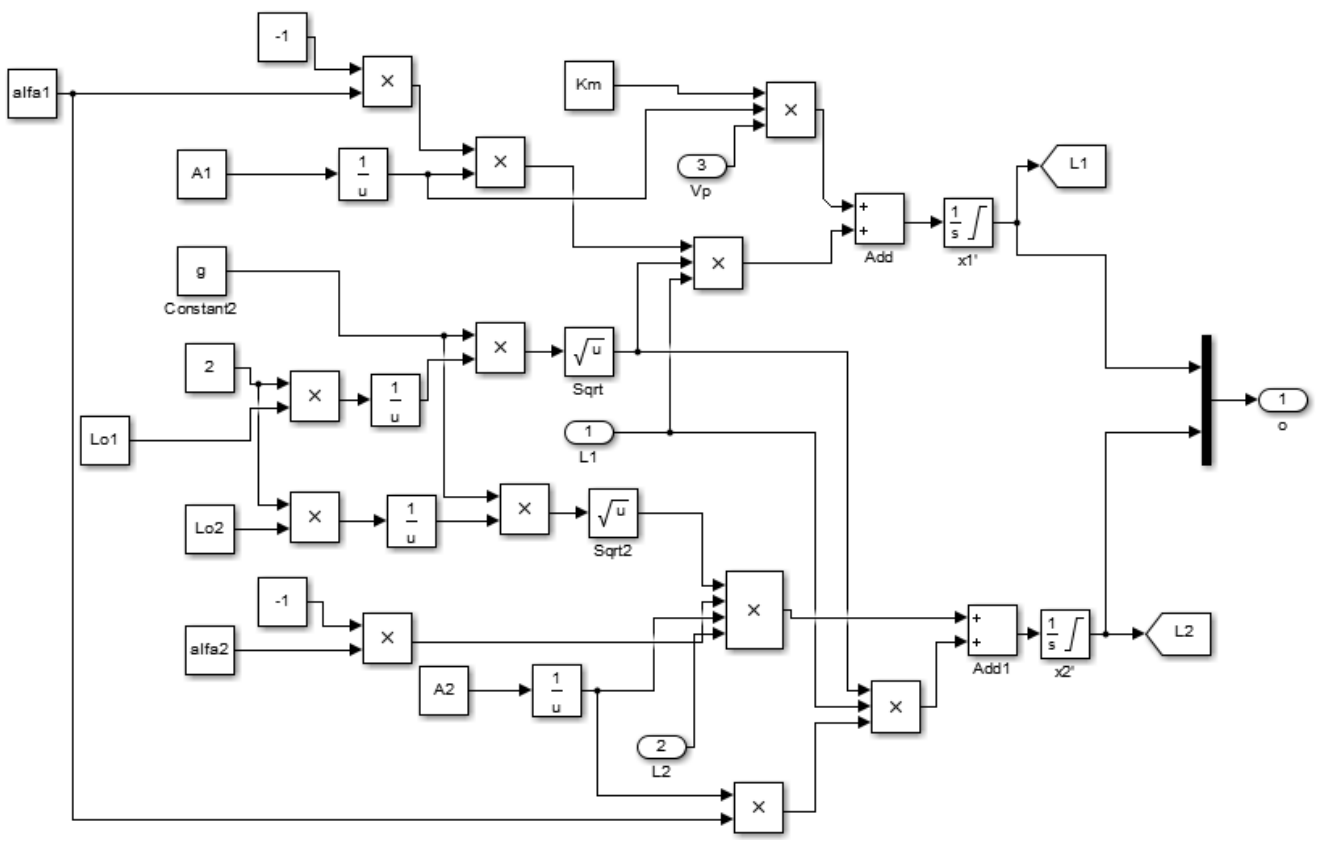

Figure 4. Design Inner of Subsystem of The Coupled Water Tank in Matlab

\subsubsection{Design of Backpropagation Neural Network}

a. Architecture of Backprogation

The back propagation neural neural in this paper is designed with an input layer, an hidden layer and an output layer, it can see at figure 5:

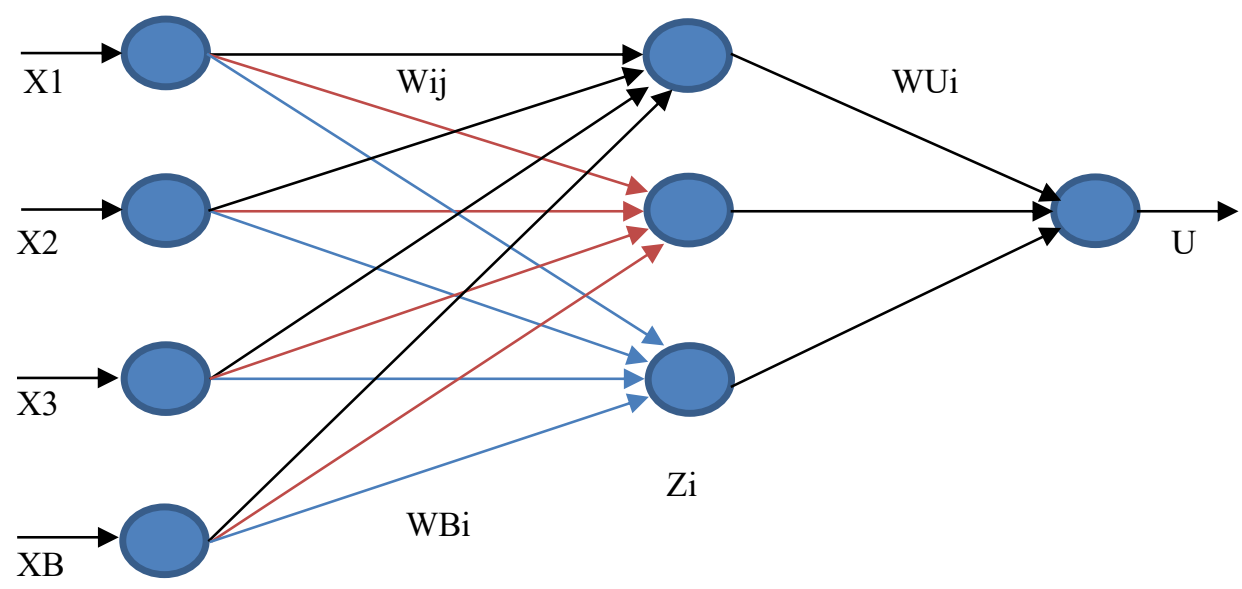

Figure 5. Architecture of Backpropagation 


\section{b. Backpropagation Algorithm}

The backpropagation neural network in this paper is designed with 1 unit input layer, 1 unit hidden layer, 1 output, and 1 bias input to hidden layer using mfile in Matlab, with the following steps:

Step 1: Determine the number of inputs, in this paper, will be used 3 inputs, because this plant is orde 2 system with $n a=2, b=1$. (na $+n b=$ number of inputs)

Step 2 : Determine the number of neurons in-unit hidden layer, in this paper, will be used 3 neurons (Number of inputs $\leq$ number of neurons in hidden layer $\leq 2$. number of inputs )

Step 3 : Initialization value of inputs, weights, learning rate constants and maximum of epoch, in this paper will be used, $\mathrm{i}=1,2,3$ and $\mathrm{j}=1,2,3$ :

a. Inputs $(X i)=\left[\begin{array}{llll}X 1 & X 2 & X 3\end{array}\right]=\left[\begin{array}{lll}e k & 0 & 0\end{array}\right]$, where error $(e k)=$ setpoint - output plant $=y \_r e f-y \_o u t$. Bias Input $(X B)=0.2$

b. Weights of input layer to the hidden layer $(W i j)=\left[\begin{array}{llllllllll}W 11 & W 21 & W H 31 & W 21 & W 22 & W 23 & W 31 & W 32\end{array}\right.$ $W 33]$, weights of input bias to the hidden layer $(W B j)=[W B 1 W B 2 W B 3]$, weights of hidden layer to the output layer $(W U i)=[W U 1 W U 2 W U 3]$ with random values between 0 to 1

c. Learning rates: for $L_{W}=0.00005, L_{W B}=0.00005, L_{W U}=0.0005, L_{U}=1$

d. Epoch: 100

e. Target output $(U Y)=0$, as long as this target is not reached, go to step 4 to 11

Fordward Calculation

Step 4 : Calculate all of output neurons in unit hidden layer:

a. Add up all of the signals output with their weight:

$$
Z H i=X B . W B i+\sum_{i j=1}^{3} X i . W i j
$$

b. Calculate the output signal using the gaussian function:

$$
Z i=e^{-(Z H i)^{2}}
$$

Step 5 : Calculate output neuron in output layer:

a. Add up the signal output with their weight:

$$
U K=\sum_{i=1}^{3} Z i . W U i
$$

b. Calculate the output signal using the linear function:

$$
U=L u \cdot U K
$$

Backward Calculation

Step 6 : Calculate the unit output error:

$$
\begin{aligned}
R U & =(\text { output of desired model }- \text { output plant })-\text { output plant } \\
& =\left(y_{-} \text {model }-y_{-} \text {out }\right)-y_{-} \text {out }
\end{aligned}
$$

and

$$
d W U j=L_{W U} \cdot \text { er } \cdot \mathrm{Zi}
$$

Step 7 : Calculate the unit hidden error:

$$
\begin{aligned}
& \text { rnetj }=R U . W i j \\
& r H j=\text { rnetj. } Z i .(1-Z i)
\end{aligned}
$$

and

$$
\begin{aligned}
& d W B j=L_{W B} \cdot \text { er.Zi.XB } \\
& d W i j=L_{W B} \cdot \text { er.Zi.Xi }
\end{aligned}
$$

Step 8 : Revision weight from hidden layer to output layer, $\mathrm{i}=1,2,3$ :

$$
W U j(\text { new })=W O j(\text { old })+d W U j
$$

Step 9 : Revision the weight from the input layer to the hidden layer:

$$
W i j(n e w)=W O i j(\text { old })+d W i j
$$

Step 10 : Revision the weight from the bias layer to the hidden layer:

Step 11 : Revision of input:

$$
W B j(n e w)=W B j(\text { old })+d W B j
$$

Where $\mathrm{i}=1$ : na- 1

$$
X X(n a-i+1)=X X(n a-1)
$$


and

$$
X X(n a+1)=U
$$

\section{RESULTS AND DISCUSSION}

In the course of testing the performance of the system we can define by steady state error, overshoots, rising time, settling time and the ability to follow setpoint changes.

\subsection{Design of Radial Basis Function Neural Network to Control Level of Coupled Water Tank}

The response output system in figure 6 , we can analisys for steady state error is $0 \mathrm{~cm}$, overshoot is $0 \%$, rising time is 48 seconds, settling time is 52 seconds, and can follow setpoint changes from 10 centimeters to 8 centimeters within 51 seconds.

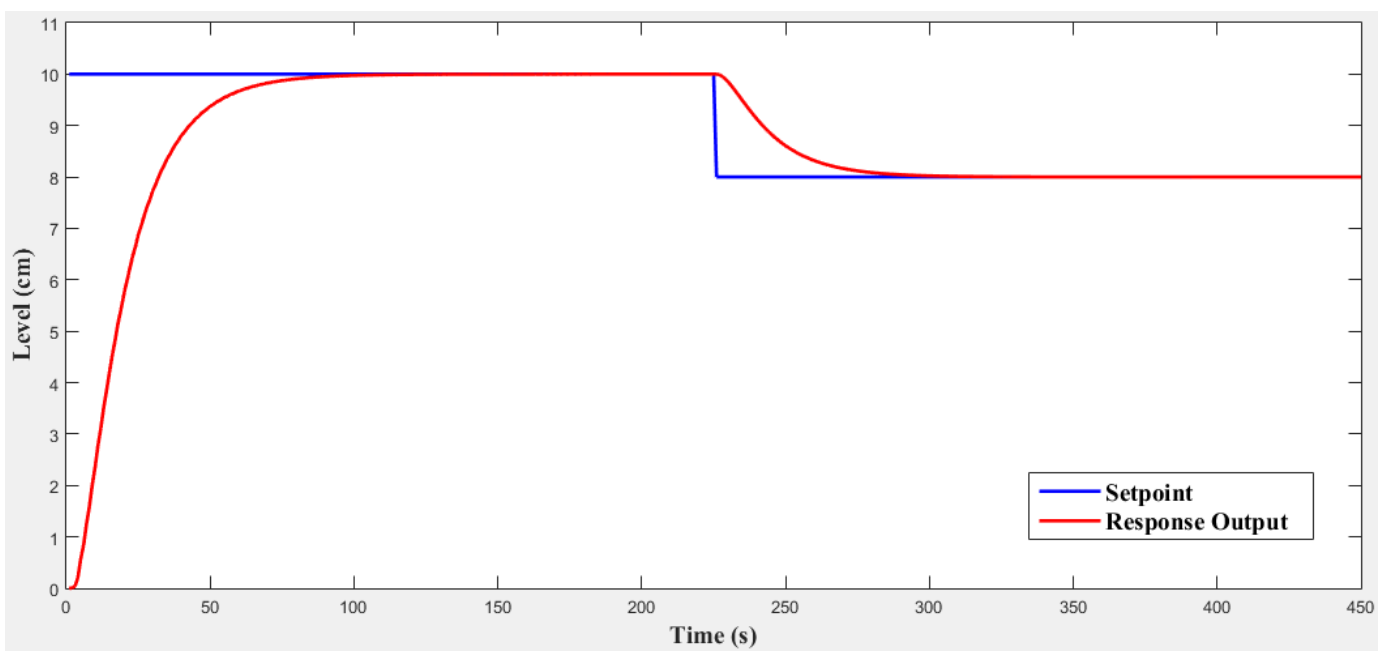

Figure 6. Response Output System for Level 2 in Tank 2 with Backprogation Neural network

\section{CONCLUSION}

The level and flow control in tanks are the heart of all chemical engineering systems. The control of liquid level in tanks and flow between tanks is a basic problem in the process industries. Many times, the liquids will be processed by chemical or mixing treatment in the tanks, but always the level of fluid in the tanks must be controlled and the flow between tanks must be regulated in presence of non-linearity. Therefore, in this paper will use neural network based on backpropagation (BP) to control of level 2 in the tank 2 with the setpoint of 10 centimeters and can follow the setpoint changes to 8 centimeters given in 225 seconds. It may be concluded from this paper, the outcome achieved from radial basis function neural network for control level 2 in tank 2 is steady-state error $=0 \mathrm{~cm}$, overshoot is $0 \%$, rising time is 49 seconds, settling time is 52 seconds and can to follow setpoint changes in 51 seconds.

\section{ACKNOWLEDGMENTS} research.

Thank you to some colleagues who have helped in completing and contributing to this

\section{REFERENCES}

[1] Konstantinos N, Charalampos G, Giannakakis, Ioannis K, Ilias S, Alex A. Nonlinear Control of a DCMotor Based on Radial Basis Function Neural Networks. International Symposium on Innovations in Intelligent Systems and Applications. Turkey. 2011; 611-615.

[2] Y. C. Liang et al., "Proper orthogonal decomposition and its application- Part II: Model reduction for MEMS dynamical analysis," J. Sound Vib., vol. 256, pp. 515-532, 2000.

[3] C. G. Looney, Pattern Recognition Using Neural Networks. NewYork: Oxford Univ. Press, 1997.

[4] K. Hornik, "Approximation capabilities of multilayer feedforward networks," Neural Netw., vol. 4, 1991.

[5] M. Leshno, V. Y. Lin, A. Pinkus, and S. Schocken, "Multilayer feedforward networks with a nonpolynomial activation function can approximate any function," Neural Netw., vol. 6, 1993.

[6] T. L. Fine and S. Mukherjee, "Parameter convergence and learning curves for neural networks," Neural 
Computat., vol. 11, pp. 747-769,1999.

[7] M. Gori and M. Maggini, “Optimal convergence of on-line backpropagation," IEEE Trans. Neural Netw., pp. 251-254, 1996.

[8] S. Mukherjee and T. L. Fine, "Online steepest descent yields weights with nonnormal limiting distribution," Neural Computat., vol. 8, pp. 1075-1084, 1996.

[9] R. Battiti, "First- and second-order methods for learning: Between steepest sescent and Newton's method," Neural Netw., vol. 4, pp. 141-166, 1992.

[10] Y. C. Liang et al., "Successive approximation training algorithm for feedforward neural networks," Neurocomput., vol. 42, pp. 311-322, 2002.

[11] A. J. Shepherd, Second-Order Methods for Neural Networks (Fast and Reliable Training Methods for Multi-Layer Perceptrons). Berlin Germany: Springer-Verlag, 1996.

[12] W. B. Liu and Y. H. Dai, "Minimization algorithms based on supervisor and searcher cooperation: Ifast and robust gradient algorithms for minminimization problems with strong noise," J. Optim. Theory Appl., vol. 111, pp. 359-379, 2001.

[13] S. W. Ellacott and D. Bose, Neural Networks—Deterministic Methods of Analysis: Thomas Computer Press, 1996.

[14] S.W. Ellacott, "The numerical analysis approach of neural networks," in Mathematical Approaches to Neural Networks, J. G. Taylor, Ed: North Holland, 1993, pp. 103-138.

[15] S. Haykin, Neural Networks, 2nd ed. Englewood Cliffs, NJ: PrenticeHall, 1999.

[16] Z. Luo, "On the convergence of the LMS algorithm with adaptive learning rate for linear feedforward networks," Neural Computat., vol. 3, pp. 226-245, 1991.

[17] P. Sollich and D. Barber, "Online learning from finite training sets and robustness to input bias," Neural Computat., vol. 10, pp. 2201-2217, 1998.

[18] W. Finnoff, "Diffusion approximations for the constant learning rate backpropagation algorithm and resistance to locol minima," Neural Computat., vol. 6, pp. 285-295, 1994.

[19] S.-H. Oh, "Improving the error backpropagation algorithm with a modified error function," IEEE Trans. Neural Netw., vol. 8, no. 3, pp. 799-803, May 1997.

[20] Z. Li, W. Wu, and Y. Tian, "Convergence of an online gradient method for feedforward neural networks with stochastic inputs," J. Computat. Appl. Math., vol. 163, no. 1, pp. 165-176, 2004.

[21] W. Wu, G. R. Feng, and X. Li, "Training multylayer perceptrons via minimization of sum of ridge functions," Adv. Computat. Math., vol. 17, pp. 331-347, 2002.

[22] Jiffy A. J, Jaffar, Riya. M. F. Modelling and Control of Coupled Tank Liquid Level System Using Backstepping Method. International Journal of Engineering Research \& Technology (IJERT). 2015; 4(6): 667-671.

[23] Abraham L, Senthilkumar, Selvakumar. Design of PI Controller Using Characteristics Ratio Assignment Method for Coupled Tank SISO Process. International Journal of Computer Application. 2011; 25(9): 49-53.

[24] Hur A, Sajjad A, Shahid Q. Sliding Mode Control Of Coupled Tank Liquid Level Control System. IEEE 10th International Conference on Frontires of Information Technology. Islamabad. 2012; 325-330.

[25] Mahyuddi N. M, Arshad R. M, Zaharuddin M. Simulation of Direct Model Reference Adaptive Control on a Coupled Tank System Using Non-linear Pant Model. International Conference on Control Instrumentation and Mechatronics Engineering. Johor. 2007; 569-576.

[26] Saad M, Albagul A, Abueejela Y. Performance Comparison between PI and MRAC for Coupled Tank Rystem. Journal of Automation and control Engineering. 2014; 2 (3): 316-321.

[27] Halim M. Comparative Study of Madani-type and Sugeno-type Fuzzy Inference System for Coupled Water Tank. Indonesian Journal of Artificial Intelligence and Data Mining (IJAIDM). 2020; 3(1): 3944. 